\title{
Le massif, ses marges et la préoccupation d'enfermement de l'habitat. Exemple des Vosges du Nord.
}

The Forest Clump, Its borders and Concerns about Forestry Advancement on Habitations. An Example from the North Vosges.

\section{Claire Labrue.}

\section{OpenEdition Journals}

Édition électronique

URL : http://journals.openedition.org/rge/2007

DOI : 10.4000/rge.2007

ISSN : 2108-6478

\section{Éditeur}

Association des géographes de l'Est

\section{Édition imprimée}

Date de publication : 1 janvier 2009

ISSN : 0035-3213

\section{Référence électronique}

Claire Labrue., « Le massif, ses marges et la préoccupation d'enfermement de l'habitat. Exemple des Vosges du Nord. », Revue Géographique de l'Est [En ligne], vol. 49 / 2-3 | 2009, mis en ligne le 08 novembre 2010, consulté le 07 septembre 2020. URL : http://journals.openedition.org/rge/2007 : DOI : https://doi.org/10.4000/rge.2007

Ce document a été généré automatiquement le 7 septembre 2020.

Tous droits réservés 


\title{
Le massif, ses marges et la préoccupation d'enfermement de l'habitat. Exemple des Vosges du Nord.
}

\author{
The Forest Clump, Its borders and Concerns about Forestry Advancement on \\ Habitations. An Example from the North Vosges.
}

Claire Labrue.

1 Il peut paraître incongru d'aborder le thème de l'enfermement lorsqu'il est question de la forêt. Ce phénomène est couramment associé à des thématiques sociales traitant des territoires fermés tels que les prisons, les asiles, les ghettos, les gated-communities (enfermements carcéral, psychiatrique, communautaire) mais jamais à des thématiques environnementales; au demeurant, la forêt est très souvent représentée comme un espace de liberté de l'imaginaire et de déplacement (Bachelard, 1957; Brinckerhoff Jackson, 2003; Tuan, 2006; Donadieu, 2007; Schama 1999). Néanmoins l'espace sylvestre n'est-il pas perçu dans le paysage comme un milieu « fermé » au contraire des autres espaces naturels, agricoles et urbains «ouverts»? Les nombreuses études parues au cours de ces quarante dernières années, et relatives aux mutations des campagnes traditionnellement vouées à l'agropastoralisme, n'ont-elles pas toutes invoqué sans exception, la «fermeture » des paysages à cause des reboisements et ses conséquences sur le vécu des habitants (Le Floch, Devanne, 2002 ; Le Floch, Devanne, Deffontaines, 2005)?

2 Si la fermeture paysagère n'est apparemment pas dotée d'une empreinte territoriale aussi forte que la fermeture sociale, il n'en reste pas moins vrai que la sylve, en raison de la vaste étendue spatiale occupée et de la hauteur de ses arbres, peut enfermer : «la fermeture [est toujours] mère de l'enfermement" (Di Méo, 2009, p. 7); la lisière, recourbée sur elle-même clôt fondamentalement l'espace, celui de la clairière. 
3 La clairière constitue un mode d'occupation humaine de l'espace immémorial et spécifique aux milieux boisés; la forêt vosgienne demeure l'archétype séculaire français de la forêt habitée. Bien qu'étant une forme fermée, la clairière traditionnelle répond à une logique d'ouverture car elle est issue du défrichement d'une partie du massif. Le terme d'enfermement semble bien inapproprié dans ce cas de figure. En réalité, ce n'est pas tant la forêt qui enferme que le rôle ou les usages que la société lui assigne ainsi que les dynamiques spatiales à l'origine de la présence de la clairière. Ainsi la forêt enferme lorsque la clairière résulte des reboisements, c'est-à-dire d'une dynamique spatio-temporelle de fermeture autour des habitations comme c'est le cas des « jeunes forêts" (Arnould, 2004) du Massif Central qui enserrent les villages dans de nouvelles clairières.

4 L'échelle du massif semble être la plus pertinente pour aborder la problématique de l'enfermement forestier. Deux raisons interdépendantes expliquent cela.

5 Tout d'abord, ce phénomène découle de l'histoire forestière de chaque région. Le vécu dans une clairière ancienne vosgienne et une clairière nouvelle de la Montagne Limousine par exemple (Labrue, 2009a et b) ne peut être identique. L'aménagement et la gestion traditionnels des espaces anciennement boisés, orientés prioritairement sur la forêt et le maintien de l'ouverture des lieux de vie, annulent le ressenti d'enfermement. Cependant aujourd'hui, même dans des régions de "vieilles forêts " (Arnould, 2004) comme celle des Vosges du Nord, ce sentiment commence à émerger. La culture forestière est "fragilisée et sur la défensive » en raison de l'évolution des logiques socio-économiques devenues urbaines "triomphante[s] et conquérante[s]» (Barthod, 2004, p. 18). Les dynamiques spatiales forestières et urbaines actuelles, redonnent alors à la forêt son pouvoir enfermant.

6 La forêt possède effectivement une forte empreinte paysagère; elle construit le paysage, déstructure les champs visuels et donne un ton et une ambiance aux lieux. Ceci n'est bien évidemment visible qu'au niveau de ses marges : en lisière, linéaire de contraste saisissant entre ouverture et fermeture, entre ombre et lumière. Ainsi le poids visuel et affectif de la forêt est tel que, lorsque les arbres sont proches de chez soi, un sentiment d'enfermement peut émerger.

\section{I - De l'ouverture traditionnelle de la clairière vosgienne à la fermeture contemporaine}

7 L'enfermement est à la fois une dynamique spatiale de rapprochement de la forêt vers l'habitat et un ressenti. Autrement formulé, l'enfermement exprime la fermeture paysagère vécue depuis l'intérieur de la forêt; il est issu du rapport sensible qu'entretient un individu avec son espace environnant (Merleau-Ponty, 1945). Qui de mieux que l'habitant pour faire l'expérience quotidienne de la proximité forestière (Tuan, 2006) et percevoir les impacts de la forêt sur son chez-soi? (Nous reviendrons sur les impacts dans la deuxième partie de cette contribution.)

8 Ce ressenti n'est pas unanime et manifeste partout et tout le temps; une approche psychosocioculturelle est nécessaire pour le comprendre et en saisir les nuances. Des enquêtes ethnographiques ont été menées auprès des habitants. Entretiens semidirectifs et questionnaires ont eu pour objectif d'établir une comparaison entre les perceptions et représentations de la forêt par les habitants de différents massifs aux 
cultures forestières différenciées (Vosges du Nord, plateau de Millevaches et Maures), de cibler les points communs et les différences entre la vision des forestiers et de ceux qui ne le sont pas, entre celle des natifs et celle des néo-ruraux ou encore celles des résidents secondaires. Concernant les Vosges du Nord proprement dites, les principaux acteurs territoriaux, l'Office National des Forêts (ONF) et le Parc Naturel des Vosges, ont été également sollicités afin de comprendre leur positionnement et actions de la gestion de l'interface habitat / forêt.

9 Au premier abord, nos enquêtes semblent démontrer qu'il est inapproprié d'appliquer le terme d'« enfermement » à la forêt vosgienne contrairement à des régions jeunes en forêt. Pourtant, en orientant nos investigations vers l'histoire des clairières vosgiennes, les modes de perception et de représentation de la forêt et plus généralement ceux de la Nature, ainsi que les outils et les pratiques en matière d'aménagement du territoire, des mécanismes de l'enfermement ainsi que des actions paysagères pour éviter son apparition, ont été et sont toujours à l'œuvre.

\section{A - Une culture forestière qui maintient la forêt à distance}

10 Pour cerner la question, à titre comparatif, une brève évocation des jeunes forêts du Massif Central est nécessaire pour comprendre la dissonance apparente entre " forêt » et « enfermement » dans les Vosges du Nord. Jusqu'à la fin du XIXe siècle, sur le Plateau de Millevaches en Montagne Limousine, la forêt était pratiquement absente; elle constituait un espace marginal, qui s'est déployé au cours du siècle dernier sous l'effet concomitant de l'exode rural et des reboisements. Ces derniers se rapprochent progressivement des secteurs habités, les enfermant dans de véritables clairières. Cette dynamique spatio-temporelle de fermeture a été suffisamment rapide pour que les habitants du Plateau se rendent compte de ce bouleversement paysager. C'est pourquoi le terme d'enfermement fait sens (compréhension et ressenti manifeste) pour les habitants et acteurs millevacois dont les systèmes de représentation du territoire du Plateau sont fondés, aujourd'hui encore, sur les souvenirs et/ou les valeurs identitaires des anciens paysages ouverts de landes.

11 Au contraire, dans les Vosges du Nord, le terme d'enfermement n'est pas du tout intégré, le mot relève du non-sens. La forêt est ancestrale et omniprésente ; la clairière vosgienne contrairement à la clairière limousine est issue des défrichements médiévaux et modernes qui répondent donc à une logique spatiale et paysagère d'ouverture. La clairière est le mode d'occupation humaine de l'espace vosgien. En définitive, habiter dans les Vosges, c'est s'enfermer inévitablement dans la forêt. Bien avant de constituer le cadre de vie des habitants, elle est avant tout le motif de l'installation des hommes en son sein : «les villages sont rarement construits dos à la forêt » mais avec la forêt comme le souligne un acteur du Parc Naturel Régional.

La forêt vosgienne est en effet une "Wald", terme allemand qui signifie à la fois le massif topographique, le massif ancien et le massif forestier, c'est-à-dire la forêt et la montagne boisée où se sont développées depuis bien longtemps des activités sylvicoles et paysannes. L'exploitation forestière avait pour objectif d'approvisionner les industries métallurgiques et les verreries situées bien souvent dans les fonds de vallées et autour desquelles se sont développés de gros bourgs comme celui de Baerenthal sur la Zinsel du Nord (principal exemple de notre étude) dès le XVIIe siècle. 
13 Pour que l'activité industrielle puisse se pérenniser, une maitrise totale du milieu a été nécessaire. En plus d'une exploitation forestière rigoureuse, des retenues d'eau ont été aménagées pour le fonctionnement des forges: "grand nombre d'étangs qui embellissent le vallon étroit dans lequel [l'usine] est construite» (Dupré, 2002, p. 7 citant Walbolck, 1907, p. 351)1. L'intérêt de ces aménagements n'était pas seulement économique, il était aussi sanitaire. Dans les vallées des Vosges du Nord, l'entretien des cours d'eau est régulier, car l'ensablement entraîne la formation de marais à l'origine de miasmes créant des conditions de vie particulièrement insalubres, accentuées par " l'important couvert forestier sembl[ant] bloquer la circulation de l'air et favoriser la formation de brouillards jugées insalubres, qui ne venaient qu'accroître les problèmes sanitaires des villageois : « fièvre des marais ", paludisme et malaria auraient provoqué plusieurs décès à Baerenthal comme à Mouterhouse. En 1833, on comptait à Baerenthal 190 cas de maladie dont 29 mortels » (Dupré, 2002, p. 9 d'après les travaux de Walbock). Assécher l'air pour le purifier fut l'obsession hygiéniste du XIXe siècle. Ces aménagements ont été tels (lavoirs, écluses, ponts, passerelles, étangs, scieries, moulins) que Baerenthal est devenue une station touristique de « cure d'air » dès 1900.

La salubrité était également favorisée par les petites exploitations des ouvrierspaysans. Le système industriel paternaliste amenait à loger les ouvriers dans des cités ouvrières collectives entourées de potagers. S'ajoutaient à cela des parcelles agricoles dans les fonds de vallées, les pentes étant laissées à la forêt. Au bord des cours d'eau, les ouvriers-paysans entretenaient des prés de fauche, récoltaient le foin nécessaire pour nourrir une ou deux vaches ou quelques chèvres que chaque ouvrier-paysan possédait pour sa propre consommation de lait. La gestion des prés était très laborieuse, mais originale et élaborée. Les parcelles étaient fauchées à la main et l'herbe souvent rapportée à dos d'homme en fin de journée pour le bétail. En effet, les prairies étant le plus souvent très humides, les animaux risquaient de s'enfoncer. Ces parcelles perpendiculaires au lit de la rivière étaient aménagées de fossés de drainage pour évacuer le trop plein d'eau qui risquait de faire pourrir l'herbe et de canaux d'irrigation pour combler le manque d'eau nécessaire à la production de fourrage et notamment du regain à la fin de l'été 2 . Ceci permettait plusieurs fauches au cours de l'été quelles que soient les conditions climatiques.

15 L'aménagement passé des fonds de vallées témoigne de façon éloquente du souci permanent du maintien de l'ouverture des espaces de vie. La clairière vosgienne représentait un espace où la nature était et devait être parfaitement domestiquée, car, comme l'affirme un habitant natif : " il faut pas que [la friche] envahisse, il faut la maîtriser ». "Propreté ", « nettoyage ", " ordre », « soigné ", " négligé " sont des termes récurrents dans le discours des acteurs et des habitants qui craignent «l'insalubrité naturelle». Ils ont souvent une perception de la nature qui les pousse à « ranger, nettoyer, présenter un territoire propre » (Piette, 2003, p. 131).

\section{$B$ - De nouveaux boisements qui créent « un certain désordre culturel » (Dupré, 2002)}

Avec les mutations socio-économiques d'après-guerre, les vallées vosgiennes comme de nombreuses campagnes françaises, n'ont ni échappé à la fermeture paysagère causées par l'enfrichement et les plantations, ni à la confusion des termes: "fermeture » et "enfermement» dans le langage courant, tant il est vrai que ces termes sont 
étymologiquement proches : «L'isolement, le début de l'encerclement par la forêt qui isole du monde extérieur, qui cache et asphyxie celui qui vit là aux yeux de la société (...) La France en friches ! C'était aussi la mort des paysans et des paysages. Ainsi est apparue une nouvelle forme de dégradation du paysage, 'sa fermeture' » (Luginbühl cité par Deuffic, 2005, p.79).

Pourtant à la lecture des travaux ethnosociologiques de Lucie Dupré traitant de la perception quotidienne de la nature dans la vallée de la Zinsel du Nord (Dupré, 2002 et 2005) et de la charte du PNR des Vosges du Nord, l'enfermement émerge de façon notoire dans ce territoire forestier. Ces documents mentionnent rarement le terme d'«enfermement». Au contraire les expressions telles que «enfrichement», « isolement ", « reboisement ", « limitation de l'espace ouvert » sont récurrentes; la «fermeture " est une préoccupation fondamentale de l'aménagement des clairières vosgiennes. Les acteurs comme les habitants ont pleinement intégré que la péjoration de la fermeture paysagère est maximale quand les boisements se retrouvent à proximité des espaces de vie, des habitations et fragmentent l'espace socialisé que représente la clairière : «Si ces friches sont au centre du village, cela donne une image de délaissé contraire à celle que l'on peut avoir d'un lieu vivant " (Charte du PNR Vosges du Nord, 2001-2011, p. 43). La fermeture devient de l'enfermement à cause de l'effet de clôture généré par la proximité des arbres (Photographie 1).

Outre l'enfrichement suite à l'abandon des parcelles traditionnellement exploitées par les paysans, des haies d'épicéas ont été plantées autour des propriétés des résidents secondaires dès les années 60 : «Parfois en bosquets, mais souvent en haies, ces épicéas marquent très fortement la propriété de celui qui les plante autour de sa résidence. En effet, ils servent de clôtures plus que symboliques et ont pour mission de protéger les habitants de toutes formes d'indiscrétions et leur fournir ainsi une intimité rêvée, signifiant ainsi leur volonté d'isolement et d'une certaine mesure -cela peut être interprétée ainsi - leur refus de s'intégrer à l'espace communal » (Dupré, 2002, p. 18). Ces habitants avaient choisi de s'enfermer au moyen d'une clôture arborée dans ce qu'ils imaginaient être la nature idyllique « la petite maison dans la prairie $»^{3}$ avec « des sapins et l'eau à la porte " (propos de l'ancien maire de Baerenthal, Labrue, 2008 ; Dupré 2002, p. 19) pour satisfaire à leur désir d'individualisme, s'isoler du territoire villageois et se soustraire de ce fait de son fonctionnement collectif hérité de la mise en valeur passée ; le système paysan des prés de fauche et la gestion de la rivière étaient en effet collectifs et réglementés ; la rivière constituait le fil conducteur et structurant du territoire de la clairière. Les reboisements, quelle que soit leur origine, témoignent du manque actuel de cohésion sociale ; " la rivière n'est plus propriétaire du village " comme le souligne ce lapsus relevé par Lucie Dupré lors d'un de ses entretiens (Dupré, 2002, p.22).

19 Aujourd'hui, ces résidences secondaires sont partiellement abandonnées. L'enfermement choisi devient subi, car "l'épicéa de bonne croissance, excède très rapidement le rôle qu'on a voulu lui faire jouer et sera paradoxalement en partie responsable de l'abandon partiel de ces habitations secondaires » (Dupré, 2002, p. 18). $\mathrm{Du}$ fait du faible temps de résidence annuelle des habitants secondaires les « rideaux d'épicéas [laissent] le champ libre aux voleurs [car] personne ne pouvait voir ce qui s'y passait » (extrait d'entretien par Dupré, 2002, p. 18). faire son retour au coeur de l'espace habité. Elle transgresse la limite de la lisière 
domaniale que ce soit par le retour de la grande faune (cerfs, sangliers, etc.), synonyme du sauvage, par une humidité ambiante créée par les effets cumulés du couvert forestier et des nombreux étangs, par la peur des «bohémiens» et des délinquants. Lorsque la forêt se densifie et se rapproche des habitations, elle réveille des peurs ancestrales ; un sentiment d'insécurité plane parce que la forêt dissimule l'Autre et ses activités prétendument suspectes (Dupré, 2002). La forêt étend son ombre sauvage (Harrison, 1992), remet en question tout l'héritage social passé, et pose la question de la maitrise de l'espace par l'homme et de sa place : « Ordre naturel que l'on impute à un certain désordre culturel » (Dupré, 2002, p. 7).

\section{C - Concurrence spatiale entre forêt et urbanisation au sein de la clairière}

21 Des actions individuelles tentent de remettre de «l'ordre culturel» par le fleurissement des maisons et jardins et par la présence de figurines servant à briser la monotonie du vert. Parallèlement, le PNR édicte des principes paysagers nécessaires à la réouverture ou à son maintien autour des espaces de vie : "rouvrir les fonds de vallées humides "; " éviter la fermeture des clairières, autant en ce qui concerne les villages que quelques fermes isolées "; "limiter les reboisements en bordure de clairières pour en éviter la fermeture progressive » (Charte PNR Vosges du Nord, p. 42 et p. 44). La mise en œuvre d'une gestion écologique des friches (GEF) est une des solutions apportées contre la fermeture. Dans les fonds de vallée sont introduits des bovins adaptés aux conditions humides : les Highland Cattles, symboles d'un nouveau mode de domestication de la nature et de réappropriation des paysages et des espaces, à rapprocher de l'opération « le paysage a du goût » qui promeut la « qualité de viande pleine nature » (site SYCOPARC ${ }^{4}$ ).

Cette nouvelle mise en valeur de l'espace, bien qu'elle s'applique à maintenir l'ouverture et "l'ordre culturel », demeure tout de même la conséquence directe de l'abandon des anciennes pratiques paysannes. La vision héritée où la nature et la forêt doivent être "propres" se confronte à une vision citadine. L'équilibre paysager traditionnel entre ouverture et fermeture est donc aujourd'hui mise à mal par une perception plus urbaine de la nature qui laisse libre cours à la biodiversité, à la friche et donc au foisonnement végétal, comme en témoigne le discours de cette nouvelle habitante qui remet en question la gestion forestière usuelle pratiquée par l'ONF : "cette rentabilité détruit l'aspect initial de la forêt "; "on maltraite la nature, on coupe des arbres tous les 2 ou 3 ans au détriment de la faune et la flore ". "L'aspect initial » est celui d'une forêt aux apparences naturelles, irrégulières, avec des bois morts favorisant la formation des écosystèmes. Au demeurant les travaux de Lucie Dupré (Dupré, 2002 et 2005) relatifs à la perception quotidienne par les habitants de l'enfrichement de la vallée de la Zinsel du Nord se sont inscrits à l'amont du processus de classement de celle-ci en zone Natura 2000.

En parallèle, la mise en place de réglementation des boisements constitue un autre outil de lutte contre la fermeture paysagère. Ainsi, à Baerenthal, la totalité de l'espace de la clairière (excepté les marges éloignées des surfaces bâties, en lisière de la forêt domaniale) qui ne relève pas du régime forestier, est interdite aux plantations privées. Les peuplements résineux actuels, une fois coupés ne pourront être replantés. Cette réglementation des boisements a été établie pour favoriser l'extension de l'espace 
ouvert, constructible, qui ne peut que s'effectuer que dans les limites strictes de la clairière. Les propos de l'ancien maire de Baerenthal sont éloquents à ce sujet : «la commune fait 3900 hectares dont 3500 hectares de forêt domaniale, on a peu de forêts privées ; il reste 400 hectares pour vivre et c'est peu. On souhaite les conserver ». Ainsi au sein de la clairière, il existe une concurrence spatiale entre urbanisation et boisements privés (Figure 1).

Figure 1. Evolution de l'occupation du sol de la clairière de Baerenthal dans la vallée de la Zinsel du Nord entre 1951 et 2007.

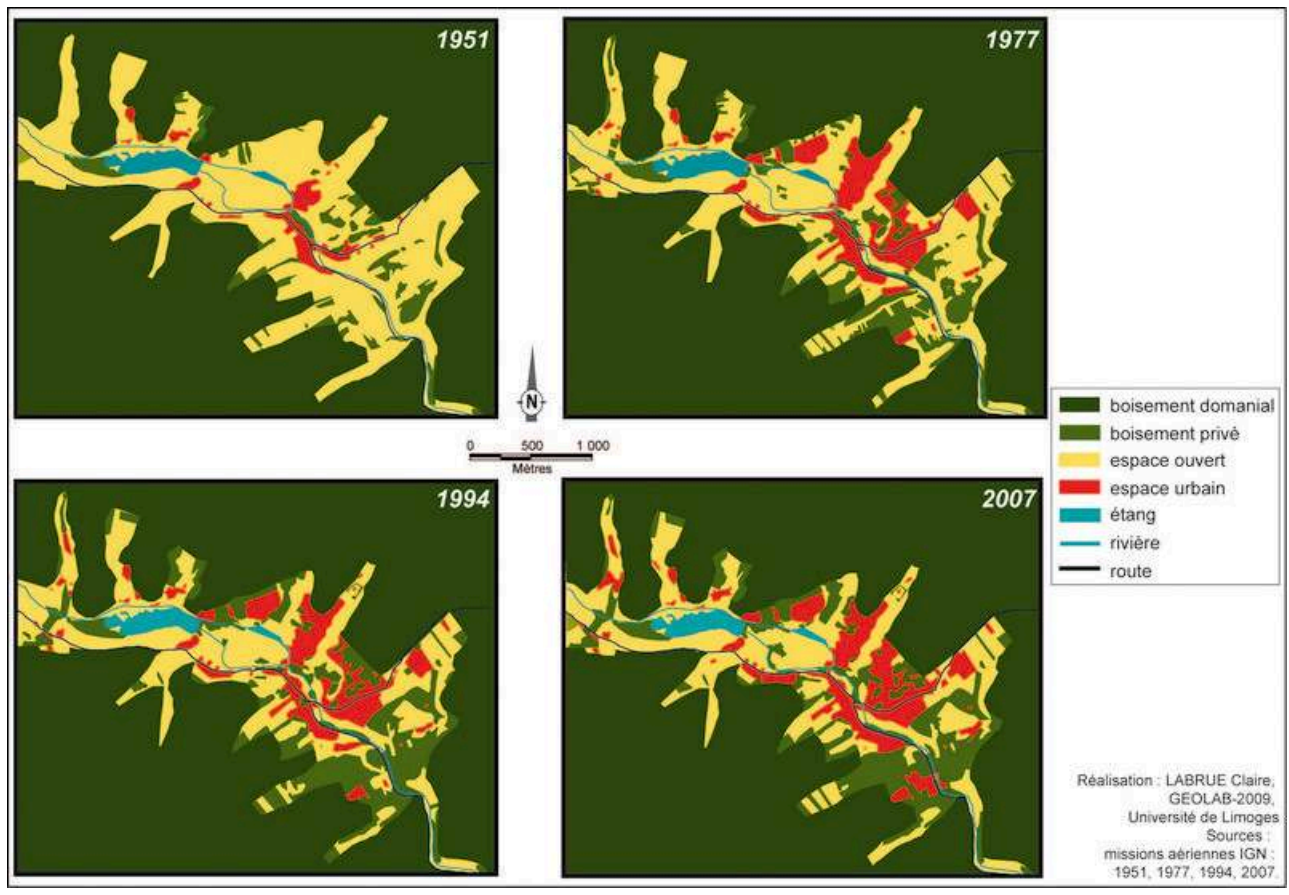

Dès 1951, la clairière se boise de façon émiettée. Les premiers boisements sont relativement éloignés des habitations, le plus souvent en lisière de forêt domaniale. En 1977, les friches et les plantations se sont multipliées et densifiées, tandis que l'espace construit s'étale. Ainsi à partir des années 90, il existe une concurrence spatiale certaine entre les boisements privés et l'urbanisation au sein de la clairière. Espace construit et espace boisé s'emboîtent l'un dans l'autre ; la clairière donne l'impression de plein.

24 L'entretien évoqué précédemment suggère que la lisière de la forêt publique demeure inamovible et même jalousement défendue aux dires d'une ingénieure de l'ONF : «le forestier défend sa limite et n'a pas envie qu'on vienne lui rogner ». Ainsi l'étalement urbain vient se heurter au «mur domanial» et comme les boisements privés et les friches, celui-ci fait ressentir aux habitants ses désagréments quotidiens. Une résidente qui vit au pied des ruines du château de Ramstein affirme clairement son « ras-le-bol » car cette forêt favorise "l'humidité »; elle note également qu' "il y a moins d'air qui circule et de la mousse » et que "les personnes âgées ont des rhumatismes». Les forestiers de l'ONF se défendent de ces inconvénients en faisant prévaloir une circulaire interministérielle $\mathrm{n}^{\circ} 77104$ du 1er août 1977 relative aux terrains boisés à conserver, qui préconise pour la délivrance d'un certificat d'urbanisme en lisière le maintien d'une «marge d'isolement entre les massifs boisés et les zones d'urbanisation, pour des raisons tenant autant à la sauvegarde des boisements qu'au bien-être, à l'ensoleillement et au sentiment de sécurité des habitants "; cette marge est équivalente à la hauteur moyenne d'un arbre mature, soit 30 mètres. 

rivière. Elles constituent les deux éléments structurant, d'une part, la vie économique et sociale du territoire et, d'autre part, les paysages par un jeu d'ouverture et de fermeture. Le paysage est le témoin visible de cet équilibre aujourd'hui mis à mal par les reboisements et l'extension de l'espace urbain vers la forêt. Bien que jamais nommé, l'enfermement est pourtant vécu ; les habitants perçoivent les deux principaux impacts paysagers de la proximité forestière qui sont à l'origine de ce sentiment : la perte des champs visuels et le manque de lumière.

\section{A. Lisibilité topographique et visibilité paysagère}

Jusqu'aux années 60, la répartition spatiale habitat, agriculture, forêt était relativement simple : une large clairière avec en son centre le bourg et les établissements industriels auréolés d'une couronne agricole, elle-même ceinturée par la forêt domaniale. La forêt étant maintenue à distance de l'habitat, l'interface était celle de l'espace forestier avec l'espace agricole. Les lieux de vie, l'empreinte humaine et la maitrise de cet espace étaient donc clairement visibles par les habitants au travers du paysage. interface avec l'habitat. Là encore cette interface spatiale se décline en plusieurs types : habitat / friches ; habitat / plantations ; habitat / forêt domaniale. Ainsi habitat et forêt s'emboîtent de manière plus complexe que par le passé. Les contours de la lisière domaniale deviennent plus flous, la linéarité de l'interface discontinue. La distribution des espaces forestiers et des espaces urbanisés paraît beaucoup plus anarchique. La perception des paysages humanisés et des grandes lignes qui les structurent est brouillée. Les boisements au sein de l'ouverture de la clairière multiplient les jeux de clair-obscur.

\section{2 - Le paysage aux marges de la forêt}

Le relief constitue le socle d'un paysage et en crée ses grandes lignes ou plutôt sa grande ligne fondatrice entre terre et ciel : l'horizon. Or la forêt peut elle aussi dessiner les principaux traits du paysage.

Le massif forestier, à l'image d'une montagne est un ensemble à très forte empreinte paysagère. Dans son dictionnaire Les mots de la géographie, Brunet définit le massif comme " une masse en relief ayant une certaine unité se distinguant ainsi des autres " (Brunet, 1992, p. 322). De grandes dimensions caractérisent la forêt. L'Inventaire Forestier National en donne la description suivante: «territoire occupant une superficie d'au moins 50 ares avec des essences forestières capables d'atteindre une hauteur supérieure à 5 mètres à maturité in situ, un couvert arboré de plus de $10 \%$ et une largeur moyenne d'au moins 20 mètres » (IFN, 2007, p. 4). Etendue arborée, hauteur des arbres et continuité du couvert végétal ${ }^{5}$ donnent à la forêt un rôle déterminant dans la construction d'un paysage et, au-delà du simple agencement, dans la construction de l'identité en raison de la place dominante qu'elle prend dans l'image renvoyée du territoire. Par exemple, dans des milieux aux modelés peu marqués, aux dénivelés assez faibles, la forêt a la capacité de déstructurer les paysages, d'homogénéiser et d'aplanir le relief. Les grandes lignes et les marqueurs spatiaux utiles à l'homme pour se repérer dans l'espace sont dissimulés sous la voûte forestière. 
La forêt est alors la seule à commander les échappées visuelles. Or la lisibilité topographique est nécessaire à l'identification d'un lieu. Celui-ci doit être visible au maximum dans son ensemble «les lieux les plus forts ne sont-ils pas ceux, [...] que le regard peut intégralement embrasser et où les repérages visuels des limites sont les plus aisés?" (Lévy, Lussault, 2003, p. 561-562) ${ }^{6}$. Traditionnellement les clairières vosgiennes du fait de leur étendue permettent cela; leurs limites sont bien visibles; elles constituent des "lieux forts ». La lisière constitue un signe visuel et symbolique tangible permettant à l'habitant de circonscrire son lieu de vie; ce linéaire arboré rompt la continuité spatiale et paysagère et crée les contrastes les plus manifestes entre ombre et lumière, entre fermeture et ouverture, entre espace habité et espace forestier.

En revanche cette force topologique de la clairière est considérablement amoindrie, quand les reboisements à proximité de l'habitat empêchent toute lisibilité topographique, et brouillent les vis-à-vis paysagers d'un versant à l'autre de la vallée. A courte distance, les mécanismes physiologiques et psychologiques de la vision forcent l'individu à d'abord percevoir la verticalité des peuplements avant leur linéarité. Le résultat de ce processus l'amène à prendre conscience de l'impact considérable des arbres dans la construction des champs d'ouverture visuelle et à réaliser, du moins en partie, l'obstruction paysagère engendrée. Dans ce contexte, les caractéristiques structurales de la végétation forestière, hauteur et linéarité, transforment la lisière en une véritable clôture des paysages que les habitants éprouvent au quotidien : " [un] sentiment d'un blocage, un envahissement rapide et inexorable procurant une sensation d'étouffement dans la mesure où la continuité visuelle est remise en question » (Dupré, 2002, p.32)

31 Les vues d'ensemble sur la clairière et le modelé de la vallée étant amoindries, la charte du PNR des Vosges du Nord préconise de « rouvrir les fonds de vallées humides. Cela paraît une action prioritaire pour redécouvrir l'unité des vallées » et ainsi « éviter la fermeture des clairières, autant en ce qui concerne les villages que quelques fermes isolées » (Charte PNR Vosges du Nord, 2001-2011, p. 42).

Photographie 1 : Vue panoramique sur le bourg de Baerenthal depuis les ruines du château de Ramstein. Cliché : LABRUE Claire, 2007.

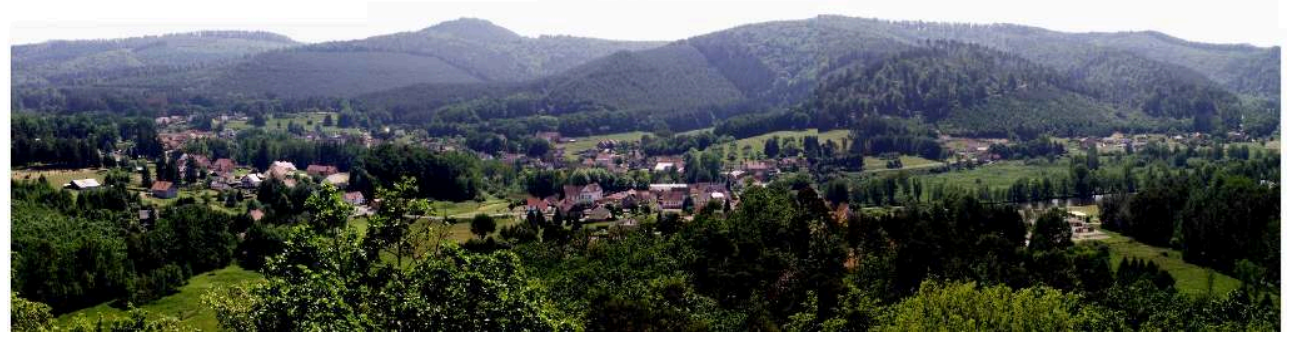

La photographie montre le bourg de Baerenthal, en fond de vallée, formant une ouverture au sein du massif forestier domanial composé essentiellement sur le versant nord d'essences résineuses. La clairière, elle-même ne constitue pas une ouverture paysagère continue. Elle est ponctuée de boisements ou de linéaires boisés privés: friches et plantations en conifères, comme nous pouvons le voir à gauche sur le cliché. A droite, nous percevons l'aménagement touristique de l'étang de Baerenthal, devenu station verte qui permet le maintien de l'ouverture.

Pour les habitahts, la vallée elle-même peut être perçue comme une "enclave » dans laquelle «on se sent parfois oppressé » aux dires d'une habitante. La forêt, et particulièrement les boisements des fonds humides accroissent donc l'enfermement 
topographique : « les pentes autour des villages, plus difficiles à cultiver que le plateau ont tendance à s'enfricher ou à être reboisées. Les villages s'enferment peu à peu, d'autant plus qu'il s'agit généralement de vallées assez étroites" (Charte du PNR Vosges du Nord, 2001 - 2011, p. 46). Le cloisonnement du relief ne permet pas originellement des vis-à-vis paysagers entre espaces habités, mais les échappatoires topographiques qu'offre la linéarité d'une vallée de part et d'autre d'un village, permettent de les relier territorialement. L'ouverture paysagère continue des principales vallées habitées est, pour les Vosgiens, un repère visible des couloirs de vie au sein du massif : « lorsque [les] friches sont à l'extérieur des villages, cela contribue à créer peu à peu des villages isolés » (Charte PNR Vosges du Nord, 2001-2011, p. 43).

\section{$B$ - Entre ombre et lumière}

33 La lisière n'est pas une simple clôture ; elle dépasse sa propre linéarité du fait de la perception que l'Homme en a et l'oppression paysagère que ce dernier ressent quand il est à proximité. La lisière intègre le "Tiers paysage" (Gilles Clément), les « délaissés » de l'aménagement des territoires qui se situent à la croisée de l'ombre et de la lumière et qui sont, de ce fait, riches de leur diversité biologique et de leurs contrastes paysagers (Clément, 2004). La lisière étend son ombre portée sur l'espace qu'elle jouxte et crée ainsi une épaisseur biogéographique (écotone) et esthétique (jeu d'ombre et de lumière) : "considérer les limites comme une épaisseur et non comme un trait " (Clément, 2004, p. 64).

34 Les délaissés ne sont pas seulement biogéographiques, ils sont également culturels ${ }^{7} \mathrm{car}$ les forêts, longtemps tenues à distance, "[ont allongé] leurs ombres dans l'imaginaire [...] 》 (Harrison, 1992, p. 157) et aujourd'hui ces ombres se matérialisent dans le réel, les clairières sont "mitées» et "noircies» par les boisements. Les contrastes entre l'espace socialisé et lumineux de la clairière, et l'espace sauvage représenté par la forêt sont absents. Ce clair-obscur ne correspond pas au schème de l'Homme en tant que " maître et possesseur de la nature... propre ». " Le soleil [qui] est avant tout le grand luminaire du Monde. Les mathématiciens en feront par la suite une masse attirante. La lumière est en haut le principe de la centralité. Elle est une si grande valeur dans la hiérarchie des images! Le monde, pour l'imagination, gravite autour d'une valeur...» (Bachelard, 1957, p. 158).

35 Les fonds de vallées connaissent inéluctablement un temps d'ensoleillement réduit du fait de l'ombre portée du relief. La forêt accentue le phénomène (Photographie 2) et détermine l'ambiance paysagère des lieux. C'est ainsi, par exemple, que les vallées souvent ponctuées de lacs peuvent être perçues de façon sinistre : «[...] l'étendue du lac prend toute la largeur de la vallée [...] presque jusqu'aux berges, s'étendent des forêt de sapins qui partagent le lac et l'enveloppent d'un noir effrayant » ( $\operatorname{Imlin}^{8}$ cité par Bloch, p.196). Au contraire les sommets où se situent bien souvent les anciens châteaux médiévaux sont pleinement exposés à la lumière : «[de] majestueux châteaux-forts en ruines trônant sur leur sommet, s'avancent au-dessus de la sombre vallée, lointains et sous une belle lumière» (Imlin cité par Bloch, 1997, p.196). Observables depuis le village, dans la vallée, les châteaux ou bien leurs ruines constituent un point de repère au milieu de la masse forestière sur lequel le regard se focalise. Comment observer ce pittoresque et ce patrimoine qui fait l'identité des Vosgiens du Nord depuis le fond de la vallée quand celui-ci est ponctué de boisements qui gênent la visibilité ? 
Photographie 2 : Vue aérienne du bourg de Baerenthal. Cliché : Roger Ruch, URL : http:// reichshoffen.pro.free.fr/rogerruch_baerenthal.jpg

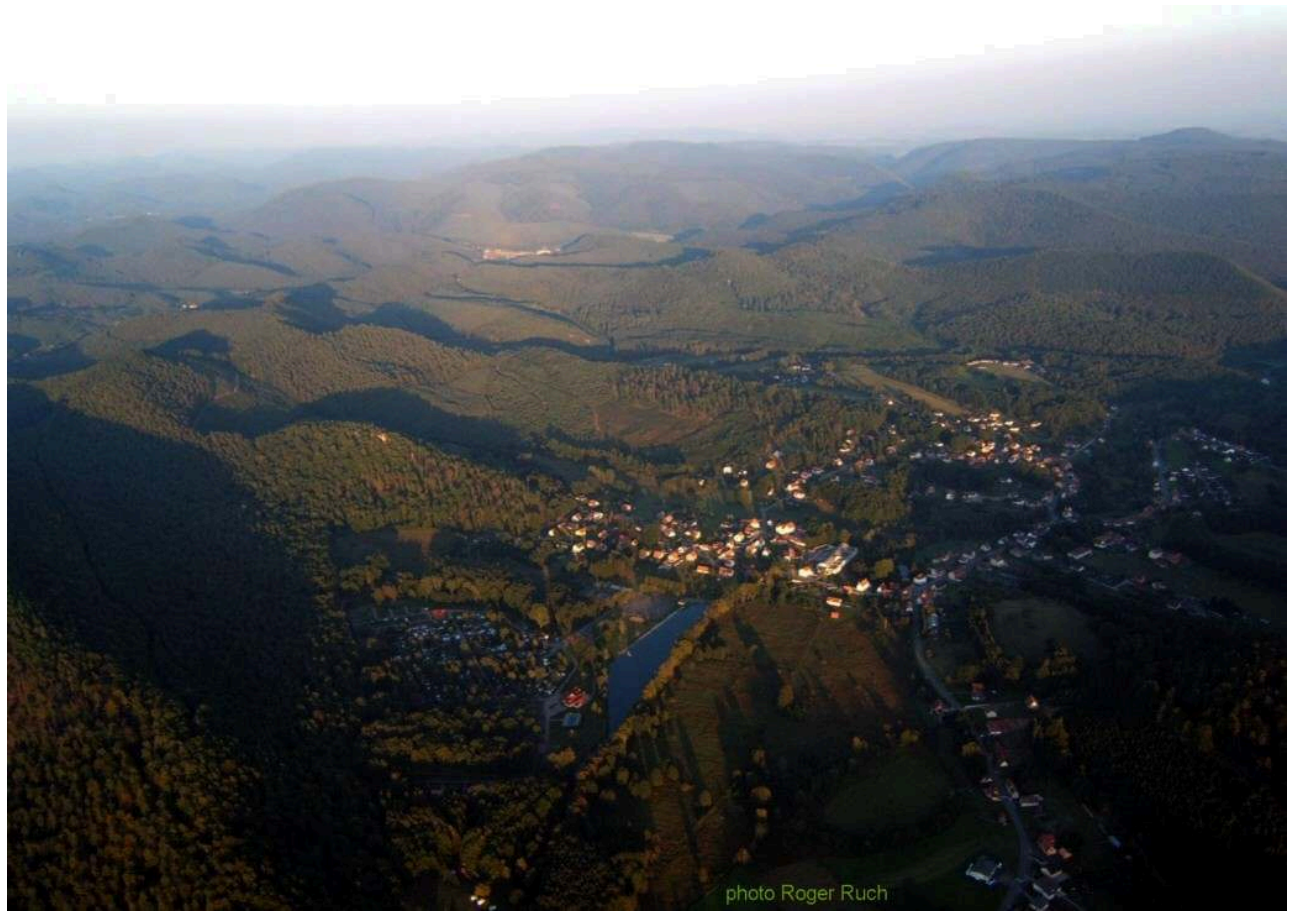

Au premier plan, se situent l'étang de dérivation de la Zinsel du Nord et son camping, à gauche de l'étang, des prés de fauche. Les boisements entre les habitations sont privés ; autour de l'espace habité, c'est le domaine de la forêt publique. Notons l'étirement des ombres portées en fin d'après-midi : celle des arbres au premier plan et celle du relief à droite de la photographie. Grâce à ce cliché ainsi que les ombres portées, nous devinons très nettement l'enfermement topographique de la vallée.

En milieu forestier, les contrastes demeurent une préoccupation majeure de l'aménagement paysager. Ombre et lumière ne se font valoir mutuellement que si elles se manifestent l'une et l'autre. La charte du PNR des Vosges du Nord souligne que les villages-clairières, qui sont autant de discontinuité paysagère au sein du massif, «constituent des points forts dans la forêt omniprésente et peuvent être particulièrement intéressants à découvrir lorsqu'on sort de la forêt ", et ajoute que "le contraste entre ces clairières ouvertes et les buttes boisées, qui les dominent, est très intéressant » (Charte PNR Vosges du Nord, 2001 - 2011, p. 44).

A plus grande échelle géographique, les arbres constituent un véritable filtre à l'ensoleillement des maisons. Une habitante affirme «compter les jours d'ensoleillement ». Si l'ombre est recherchée en été pour sa fraîcheur, dans certaines circonstances elle est problématique, particulièrement en hiver. L'ensoleillement hivernal est limité et l'ombre portée des arbres accentue le phénomène. En outre, elle maintient l'humidité, la neige et le gel. Elle peut éventuellement perturber le bon fonctionnement des panneaux solaires.

L'étirement de l'ombre portée dépend de plusieurs facteurs : en premier lieu celui de la hauteur des arbres et de l'opacité du peuplement forestier, sempervirent ou caduc, mais également de la course apparente du soleil (d'est en ouest), c'est-à-dire de l'angle d'incidence des rayons solaires au sol. L'obliquité du rayonnement solaire varie selon l'heure de la journée, la saison, la topographie ; pente, exposition et implantation de la forêt par rapport aux maisons commandent aussi l'éclairement des sites habités. Il est 
évident, par exemple, qu'une forêt au feuillage persistant implantée au sud d'une maison, c'est-à-dire dans l'axe d'arrivée du rayonnement solaire est plus gênante qu'une même forêt implantée au nord. La forêt implantée au sud sera bien davantage ressentie comme enfermante, à cause de l'ombre et du manque de lumière qu'elle génère au cours de la journée, que celle du nord, perçue comme une protection contre les vents froids (Figure 2).

Figure 2 : Modélisation de l'étirement de l'ombre portée d'une jeune lisière de douglas ( $15 \mathrm{~m}$ de haut) implantée au sud d'une habitation à différentes heures et journées de l'année.

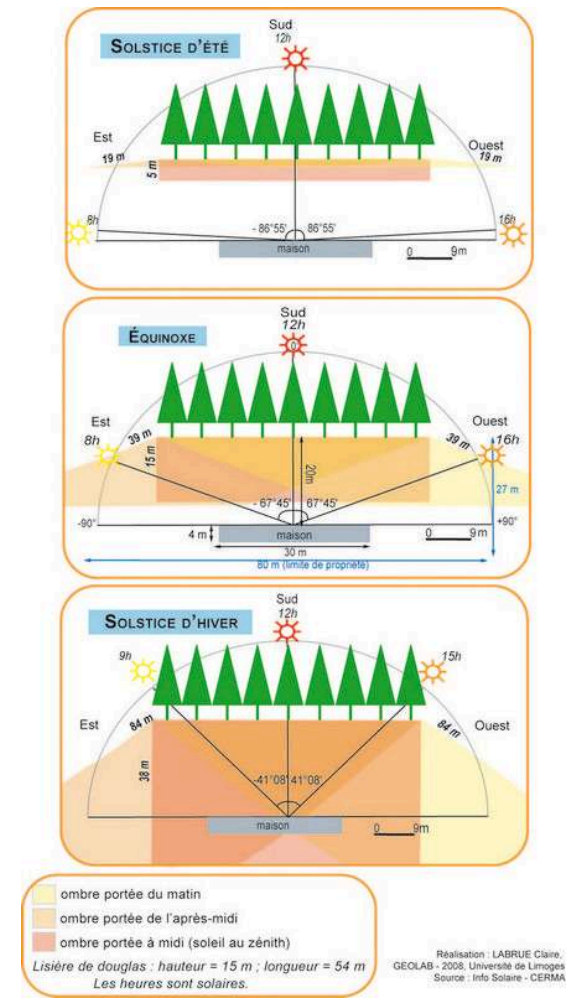

Cet exemple a été établi à partir d'une interface réelle entre une maison et la forêt en Montagne Limousine.

\section{C - De la forêt écrin à la forêt écran}

L'exemple vosgien montre la complexité du regard porté par les habitants sur la forêt. Une nouvelle fois, la vision traditionnelle rurale de la forêt et la vision contemporaine urbaine se confrontent. Sur la page d'accueil du site de la commune de Baerenthal, il est possible de lire ce texte très éloquent mêlant les deux visions : «Aujourd'hui, ce ne sont plus des ours mais des cerfs, des biches, des chevreuils, des sangliers et des renards qu'on rencontre dans la forêt omniprésente, qui forme un écrin de verdure tout autour du village et qui incite à la randonnée $»^{9}$. Les animaux donnent à la forêt son caractère sauvage qui attire. Or cette attirance est sécurisée par la gestion forestière rationnelle de l'office National des Forêts et aussi par l'aménagement des pistes de randonnées balisées du Club Vosgien.

La perception par les habitants transforme donc la forêt en un écran ou un écrin de verdure. Deux grandes variables temporelles en sont à l'origine : celle de la saison et celle de la culture. A des degrés divers, et selon le temps de résidence à proximité de la 
forêt et l'histoire individuelle de chaque habitant, l'appréciation de la forêt fluctue. Les résidents secondaires ont, dans un premier temps, vu dans les haies d'épicéas un écrin de verdure et un moyen de s'isoler dans une nature; puis, dans un deuxième temps, se sont aperçus de l'effet écran de ces arbres au fur et à mesure de leur croissance : « au début on est content puis ça pèse [...] cette verdure envahissante ». Les natifs, eux, voient en ces nouveaux boisements un écran à la vie villageoise et à la mémoire du passé, alors que les nouveaux habitants y perçoivent une réserve écologique : «Une nature spontanée refait donc son apparition de façon perceptible pour les habitants. Mais le contexte socio-économique favorable à ce retour de la nature se heurte à des réticences culturelles. En effet, là où les naturalistes voient un retour bénéfique de la nature, certains habitants des Vosges du Nord voient un recul des paysages domestiqués et une avancée du sauvage qui les inquiètent (Dupré, 2002). En forêt, malgré des évolutions certaines, les forestiers restent attachés à un contrôle social de la forêt et à une logique de travaux (Piette, 2003) » (Génot, Duchamp, Morelle, 2005).

41 Le passage d'écran à écrin peut se mesurer au moyen des contraintes et des aménités paysagères de la forêt. Dans les Vosges du Nord, la forêt est perçue comme un écran lorsqu'elle renforce l'enfermement topographique des vallées, diminue la portée des champs visuels et amplifie l'ombre portée du relief. Un village entouré de forêt sera considéré comme un écrin de verdure seulement si les cônes de visibilité sont suffisamment larges pour que les habitants ne se sentent pas engloutis par la végétation forestière. La surface d'ouverture de la clairière doit être alors suffisamment étendue pour que les habitants puissent se situer dans l'espace et s'inscrire pleinement sur le territoire dans lequel ils vivent. La lisière ne doit pas être perçue comme un mur de "végétation complètement dense " et ombrageux donnant le sentiment d'être dominé par la « nature » forestière.

\section{Conclusion}

La problématique de l'enfermement s'insère tout à fait dans une réflexion à l'échelle du massif forestier. Celui-ci représente une échelle pertinente pour étudier ce phénomène. En effet celui-ci apparaît sur ses marges internes, c'est-à-dire au niveau des clairières qui constituent autant de discontinuités spatiales et de ruptures paysagères du massif. En outre, le ressenti d'enfermement dépend étroitement du niveau d'assimilation culturelle de la forêt par les habitants et des pratiques de gestion qui ont contribué durant des siècles à forger l'identité du territoire concerné. Dans les Vosges du Nord, les pratiques de gestion et d'aménagement du territoire avaient pour principal objectif de maintenir l'ouverture des clairières afin d'éviter justement les désagréments de la proximité forestière et l'enfermement, matériel comme sensible, ce qui explique pourquoi l'enfermement forestier ne parait pas manifeste de prime abord dans la région.

43 Aujourd'hui habitat et forêt sont proches, mais il existe une distance culturelle entre ces deux ensembles du fait de l'urbanisation des modes de vie, des nouveaux modes de représentations de la nature et des nouveaux usages de la forêt se limitant à la simple récréation. C'est dans cet apparent paradoxe, rapprochement physique et éloignement culturel, qu'est le vivier de l'enfermement, car c'est avant tout un vécu qui sollicite bien moins l'esthétique du paysage que l'esthésie, c'est-à-dire les sensations que procure le paysage forestier proche. La forêt du fait de sa morphologie marque les paysages d'une 
très forte empreinte : l'habitant ne peut donc être insensible à cette masse forestière, plus encore quand elle est proche de son lieu de vie, de son habitat.

\section{BIBLIOGRAPHIE}

Arnould P. (2004) - « Nouvelles forêts, vieilles forêts, forêts de l'entre-deux (XIXe et XXe siècles) : rationalité économique et fertilité symbolique ", in Les forêts occidentales du Moyen-Âge à nos jours, Actes des XXIVe Journées Internationales d'Histoire de l'Abbaye de Flaran, septembre 2002, Toulouse, Presses universitaires du Mirail, A. Corvol-Dessert / Editions, 302 p., p. 253-277.

Association Espaces et Recherches (1999). - La forêt, livret-guide nature, milieux naturels originaux des Vosges du Nord, PNR Vosges du Nord, 33 p.

Bachelard G. (1957) - La poétique de l'espace, Paris, PUF, 9e édition 2008, Quadrige, 215 p.

Barthod C. (2004) - « Protection de la nature et identités culturelles en France », History, culture and conservation, Policy Matters, p. 18-26

URL : http://www.iucn.org/themes/ceesp/Publications/newsletter/PM13-Section1-

part1.pdf

Bloch A. (1997) - « Le pittoresque et les Vosges. Paysages romantiques des Vosges du Nord » p 193 à 204, in Groupe d'Histoire des Forêts Françaises, La Forêt. Perceptions et représentations, Paris, L'Harmattan, $401 \mathrm{p}$.

Brinckerhoff Jackson J. (2003) - À la découverte du paysage vernaculaire, (titre original : Discovering the vernacular landscape, 1984) Arles, Actes Sud, ENS Paysage, $278 \mathrm{p}$.

Brunet R., Ferras R., Théry H. (1992) - Les mots de la géographie. Dictionnaire critique. Paris, RECLUS- La Documentation Française, 518 p.

Clément G. (2004) - Manifeste du Tiers paysage, Paris, Editions Sujet Objet, Collection L'Autre Fable, $69 \mathrm{p}$.

Deuffic P. (2005) - « La fermeture des paysages dans le massif central : regards d'habitants sur une question d'experts », Cahiers d'économie et sociologie rurales, numéro 75, p.75-96.

Di Méo G. (2009) - « Espaces d'enfermement, espaces clos : l'esquisse d'une problématique », in Doc'Géo, Espaces d'enfermement, espaces clos, actes de colloque de la Journée Doc'Géo du 20 mai 2008, Bordeaux, Cahiers ADES, n4 2009, 161 p., p. 7-18.

Donadieu P. (2007), La demande sociale et l'offre d'espaces ouverts dans les régions urbaines,10 p. URL : www.bergerie-nationale.educagri.fr

Dupré L. (2002). - La construction sociale de la nature ordinaire. Expertise ethnosociologique dans la vallée de la Zinsel du Nord, Rapport pour le Parc naturel régional des Vosges du Nord, 97 p.

Dupré L. (2005). - « Des friches : le désordre social de la nature », Terrain, revue de l'ethnologie de l'Europe, numéro 44, p. 125-136. 
Génot J-C., Duchamp L., Morelle S. (2005) - Place de la naturalité dans le Parc naturel régional des Vosges du Nord, réserve de Biosphère.

http://www.mab-france.org/fr/publi/naturalite_territoires/GENOTetal.htm

Harrison R. (1992) - Forêts. Essai sur l'imaginaire occidental (titre original : Forests : the Shadow of Civilization, 1992), Paris, Champs, Flammarion, $406 \mathrm{p}$.

IFN (2007) - La forêt française. Les résultats issus des campagnes d'inventaire 2005 et 2006, 142 p.

Labrue C. (2010). - « La forêt comme clôture : l'enfermement de l'habitat par la forêt », in Projets de paysage, ENSP, à paraître en ligne (juin).

Labrue C. (2009a). - L'enfermement de l'habitat par la forêt. Exemples du Plateau de Millevaches, des Maures et des Vosges du Nord, thèse de doctorat en géographie, Limoges, $490 \mathrm{p}$.

Labrue C. (2009b). - « L'enfermement des habitations par la forêt. Exemple du Plateau de Millevaches ». In Doc'Géo, Espaces d'enfermement, espaces clos, actes de colloque de la Journée Doc'Géo du 20 mai 2008, Cahiers ADES n4, 161 p. ; pp.125-138.

Le Floch S., Devanne A.-S. , Deffontaines J.-P. - (2005), « La "fermeture du paysage" : au-delà du phénomène, petite chronique d'une construction sociale ", L'Espace Géographique, 2005 - 1, p. 49-64.

Le Floch S., Devanne A.-S. (2002) - La Notion de fermeture du paysage : trente années de succès sur la scène institutionnelle française, Paris, Cemagref, Bureau des Paysages (MATE/DNP), 30 p. + annexes.

Lévy J., Lussault M. (sous la dir.) (2003) - Dictionnaire de la géographie et de l'espace des sociétés, Paris, Belin, 1034 p.

Merleau-Ponty M. - (1945), Phénoménologie de la perception, édition de 2005, Paris, Gallimard, p. 537.

Parc naturel régional des Vosges du Nord - Charte 2001-2011, annexes, plan du parc.

Piette S. (2003). - « Réflexions sur la reconstitution des forêts après la tempête de 1999 dans le Parc naturel des Vosges du Nord ", Annales scientifiques de la Réserve de Biosphère transfrontalière, tome 11, p.121-146.

Schama S. (1999). - Le paysage et la mémoire. Paris, Seuil, 720 p.

Tuan Y.-F. (2006) - Espace et Lieu. La Perspective de l'Expérience (titre original : Space and Place. The Perspective of Experience, University of Minnesota, USA, 1977), Paris, Folio, collection Archigraphy Paysages, $219 \mathrm{p}$.

\section{NOTES}

1. Walbock L.-G. (1907) - Monographie d'une usine lorraine. Mouterhouse depuis 1614 jusqu'à 1900, Jahrbuch d. ges. F. lothr Geschichte u. Altertumks, p. 347-390.

2. P. HAMANN, "Les fonds de vallées dans le Parc naturel régional des Vosges $d u$ nord", diaporama. URL : http://www.ac-strasbourg.fr/sections/ enseignements/secondaire/pedagogie/actions_educatives/education_a_lenviro/ aides_pour_elaborer/outils_pedagogiques/les_fonds_de_vallee/view

3. Les résidences secondaires ont été construites sans permis; le Plan d'Occupation des Sols de Baerenthal a été élaboré à la fin des années 70. 
4. URL : www.parc-vosges-nord.fr

5. Du moins, pour les forêts tempérées.

6. Définition de «lieu»

7. Nous apportons un complément à la définition de Gilles Clément du Tiers paysage en affirmant qu'il ne correspond pas seulement aux espaces refuges de la biodiversité (où la nature est livrée à elle-même) mais aussi aux espaces que les Hommes ne savent plus gérer, du fait des nouveaux usages de la forêt, et de l'espace rural en général qui se réduit bien souvent et seulement à son usage résidentiel.

8. IMLIN E.F., Vogesische Ruinen und Natürshonheiten, 1815-1821, ouvrage illustré de quatorze vues, gravure sur cuivre.

9. www.baerenthal.eu/fr/index_fr.html

\section{RÉSUMÉS}

Les Vosges du Nord n'ont pas échappé au déclin des activités industrielles et paysannes au cours $\mathrm{du}$ XXe siècle. Aux marges internes du massif, les clairières, lieux de vie par excellence, se sont reboisées. Nées des défrichements d'une partie du massif et maintenues ouvertes par une auréole agricole autour du bourg, les clairières enregistrent aujourd'hui une dynamique de fermeture des paysages. Parallèlement, elles connaissent une extension de leur espace construit, si bien qu'aujourd'hui l'interface entre espace habité et espace forestier est plus complexe que par le passé. Le rapprochement réciproque des maisons et des arbres fait prendre conscience aux habitants de l'effet clôturant de la lisière, qui, au quotidien, peut se traduire en un véritable sentiment d'enfermement.

In the twentieth century, the North Vosges were not exempt from industrial and farming decline. On the inner borders of the forest clump, the glades, a living place par excellence, have been reforested. The glades were generated by forest clearing; the agricultural activities around the burg kept them open. But today, the countryside of these glades is in the process of closing in on the forest. At the same time, they have been effected by urban spreading, to the extent that the interface between urban and forestry spaces is more complicated than days of old. The closeness of houses and trees makes inhabitants realize the closing in effect of the forest's outskirts, which could result in a real feeling of confinement in daily life.

\section{INDEX}

Mots-clés : forêt, habitat, enfermement, paysage, clairière

Keywords : forest, habitat, confinement, landscape, glade 
AUTEUR

CLAIRE LABRUE.

UMR 6042 Géolab. Faculté des Lettres et Sciences Humaines. 39 E, rue Camille Guérin 87036

Limoges Cédex. claire.labrue/CHEZ/unilim.fr 